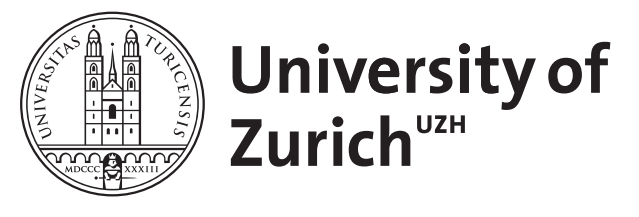

\title{
Burning cars in a parking lot
}

\author{
Bertoin, J
}

\begin{abstract}
Knuth's parking scheme is a model in computer science for hashing with linear probing. One may imagine a circular parking lot with $\mathrm{n}$ sites; cars arrive at each site with unit rate. When a car arrives at a vacant site, it parks there; otherwise it turns clockwise and parks at the first vacant site which is found. We incorporate fires into this model by throwing Molotov cocktails on each site at a smaller rate $\mathrm{n}-$, where $0<<1$ is a fixed parameter. When a car is hit by a Molotov cocktail, it burns and the fire propagates to the entire occupied interval which turns vacant. We show that with high probability when $\mathrm{n} \rightarrow \infty$, the parking lot becomes saturated at a time close to 1 (i.e. as in the absence of fire) for $>2 / 3$, whereas for $<2 / 3$, the average occupation approaches 1 at time 1 but then quickly drops to 0 before the parking lot is ever saturated. Our study relies on asymptotics for the occupation of the parking lot without fires in certain regimes which may be of independent interest.
\end{abstract}

DOI: https://doi.org/10.1007/s00220-011-1288-8

Posted at the Zurich Open Repository and Archive, University of Zurich

ZORA URL: https://doi.org/10.5167/uzh-77147

Journal Article

Presentation

Originally published at:

Bertoin, J (2011). Burning cars in a parking lot. Communications in Mathematical Physics, 306(1):261290.

DOI: https://doi.org/10.1007/s00220-011-1288-8 


\title{
Burning cars in a parking lot
}

\author{
Jean BERTOIN
}

University of Zürich 
Forest fires introduced by Drossel and Schwabl (1992). 
Forest fires introduced by Drossel and Schwabl (1992).

Sites of a lattice are either vacant or occupied by a tree.

Each vacant site becomes occupied at unit rate, independently of others sites

(imagine that seeds are sown uniformly at random). 
Forest fires introduced by Drossel and Schwabl (1992).

Sites of a lattice are either vacant or occupied by a tree.

Each vacant site becomes occupied at unit rate, independently of others sites

(imagine that seeds are sown uniformly at random).

The connected components of occupied sites are viewed as forests. 


$0-0-0-0-0-0-0$




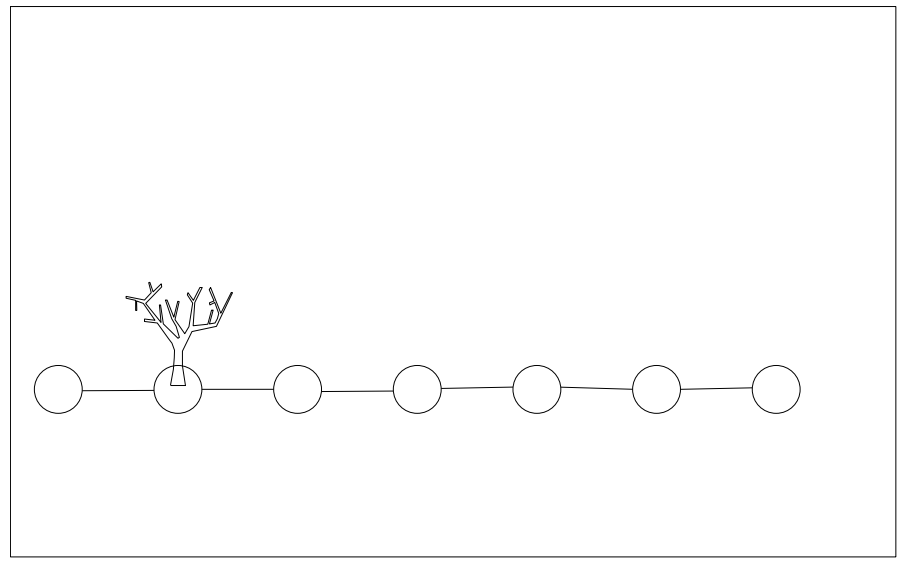




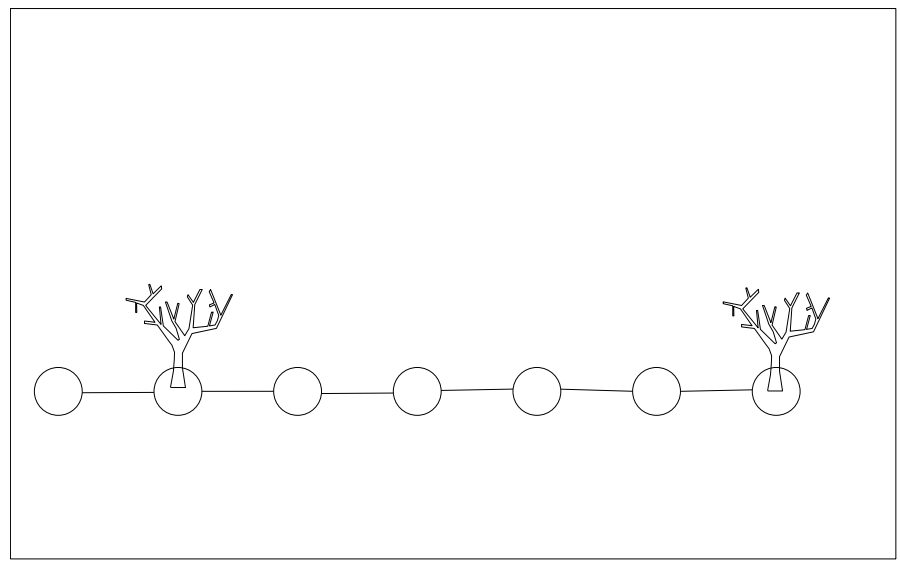




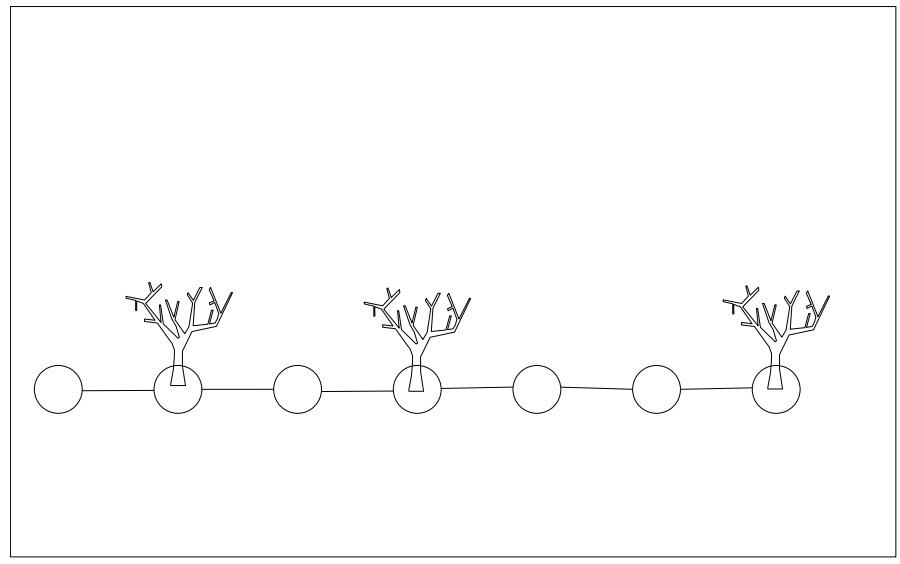




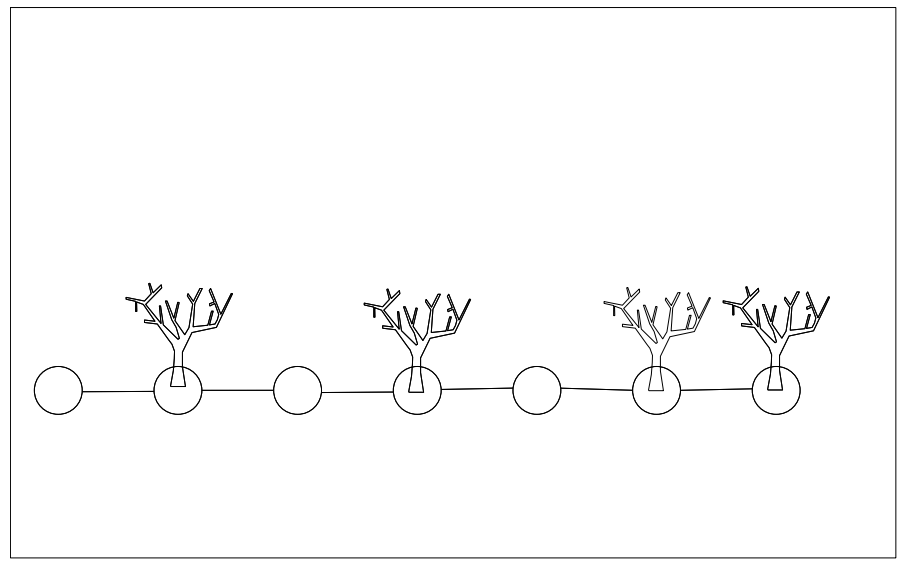




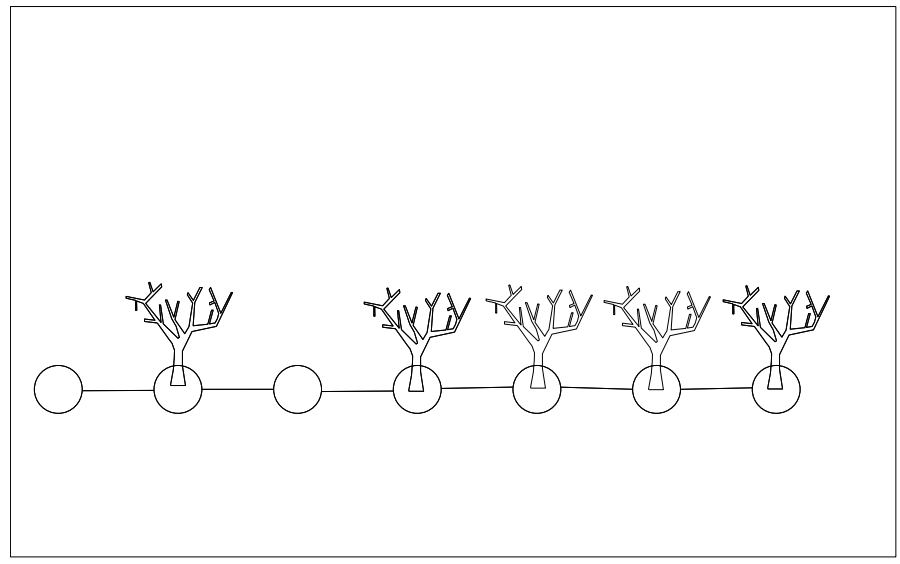


Furthermore, each tree can also be hit by a lightning at some rate $\lambda>0$. 
Furthermore, each tree can also be hit by a lightning at some rate $\lambda>0$.

Then the tree burns and the fire propagates to the entire forest.

Every site connected to the site hit by a lightning becomes instantaneously vacant. 


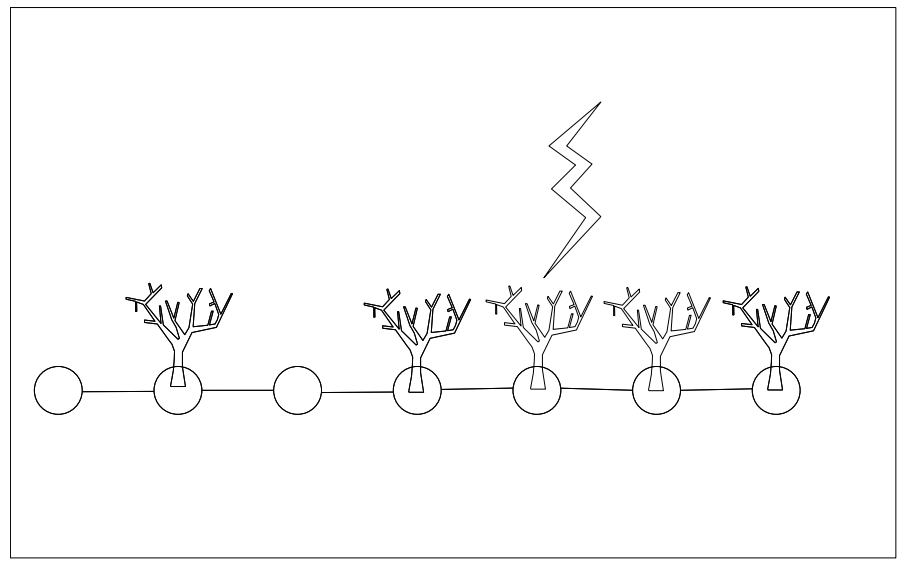




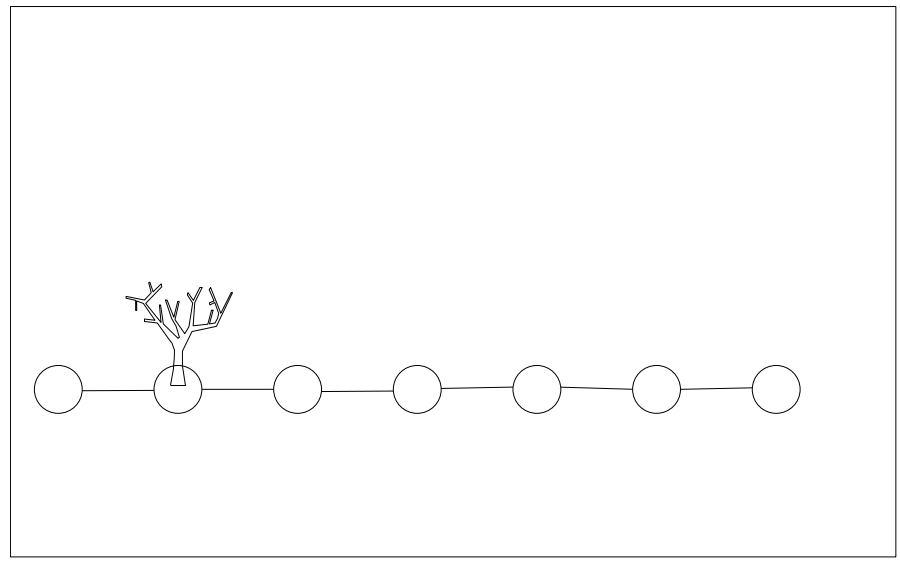




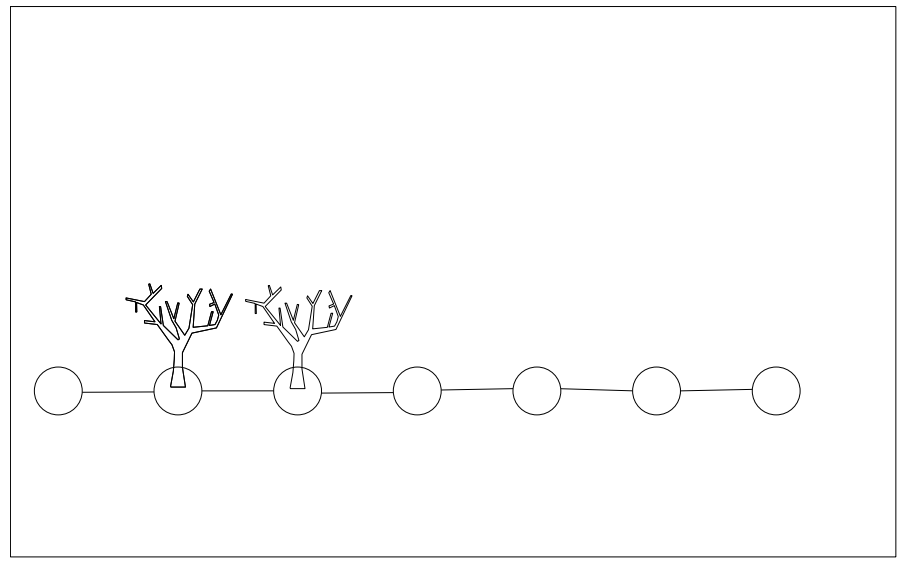




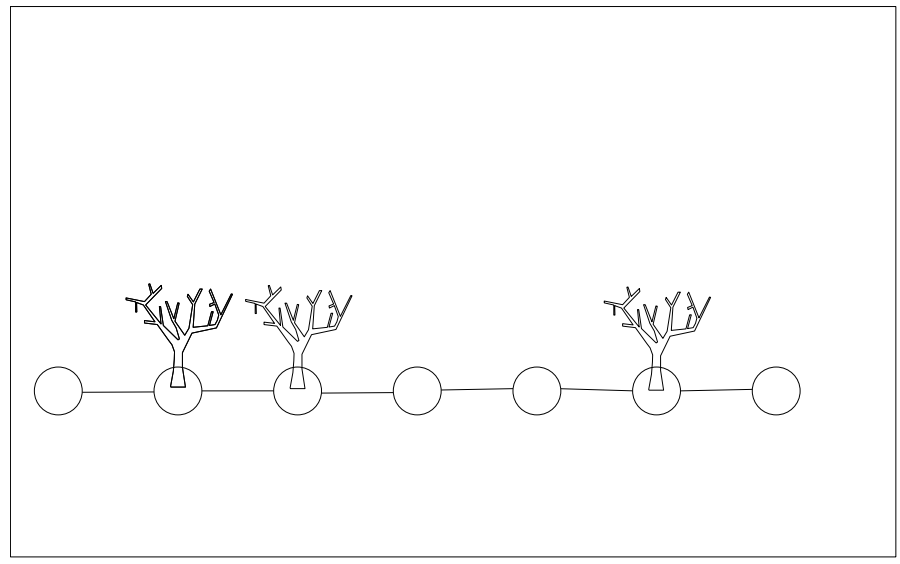




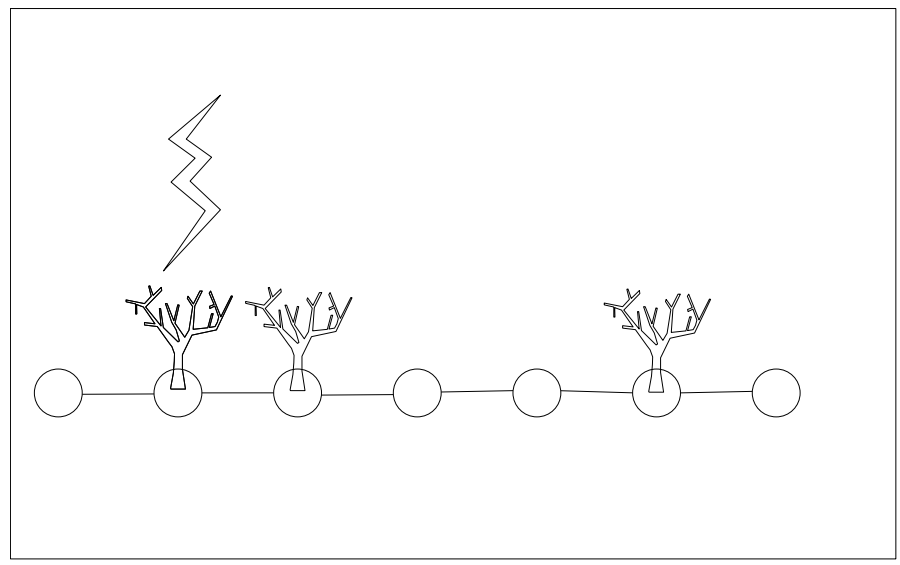




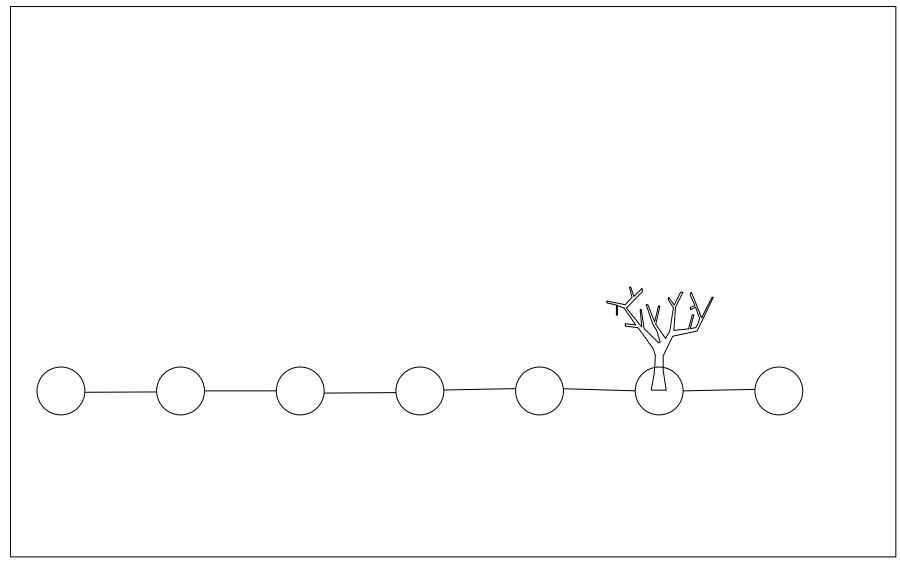




\section{The evolution alternates growth/destruction.}




\section{The evolution alternates growth/destruction.}

\section{Forests grow :}


The evolution alternates growth/destruction.

\section{Forests grow :}

- by addition of trees on their boundaries 
The evolution alternates growth/destruction.

\section{Forests grow :}

- by addition of trees on their boundaries

- by merging when a vacant site separating two forests turns occupied. 
The evolution alternates growth/destruction.

Forests grow :

- by addition of trees on their boundaries

- by merging when a vacant site separating two forests turns occupied.

Forests disappear when hit by a lightning. 
The evolution alternates growth/destruction.

Forests grow :

- by addition of trees on their boundaries

- by merging when a vacant site separating two forests turns occupied.

Forests disappear when hit by a lightning.

The largest the forest grows, the more flammable it becomes. This is the source of self-organized criticality. 
The evolution alternates growth/destruction.

Forests grow :

- by addition of trees on their boundaries

- by merging when a vacant site separating two forests turns occupied.

Forests disappear when hit by a lightning.

The largest the forest grows, the more flammable it becomes.

This is the source of self-organized criticality.

Recent mathematical studies by van den Berg, Brouwer, Járai, Dürre, Bressaud \& Fournier, Ráth \& Tóth, ... 
Knuth's parking scheme is a model for hashing with linear probing (Computer Science).

Think of $\mathbb{Z} / n \mathbb{Z}$ as a circular parking where initially all sites are vacant.

Cars arrive one after the other, uniformly at random. 


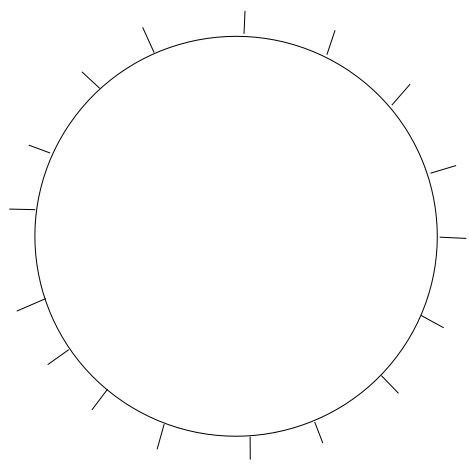




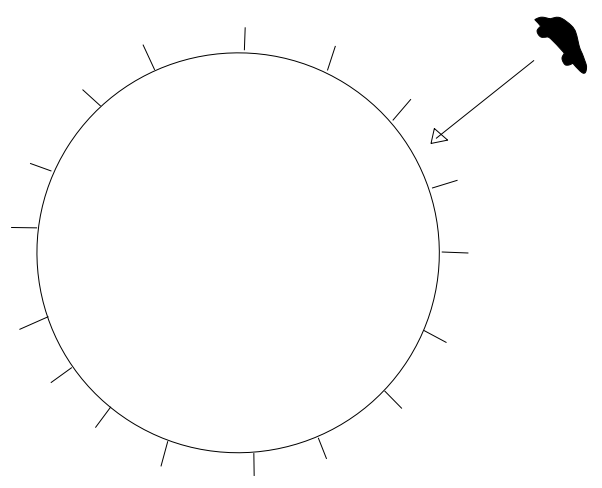

$4 \square>4 \square>$ 
Introduction

Main results

How many cars have burnt?

Formation of mesoscopic blocks
Forest fires

Knuth's parking scheme

Fires in the parking scheme

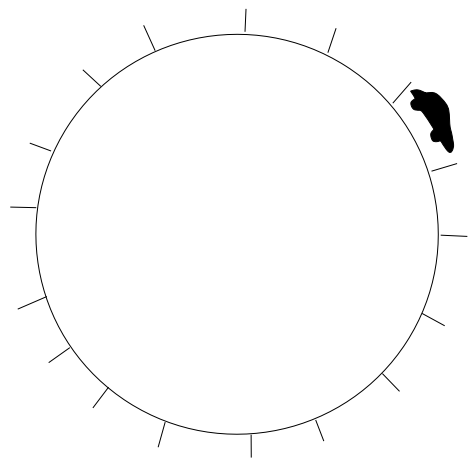

Jean BERTOIN

Burning cars in a parking lot 


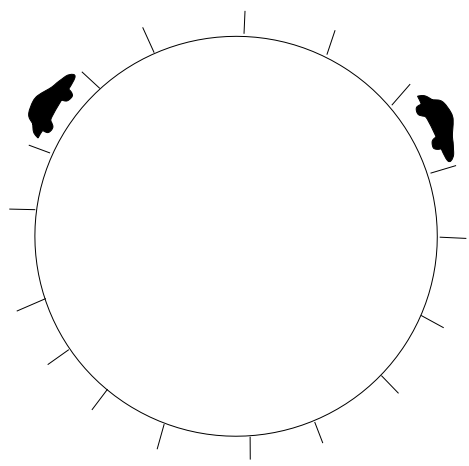




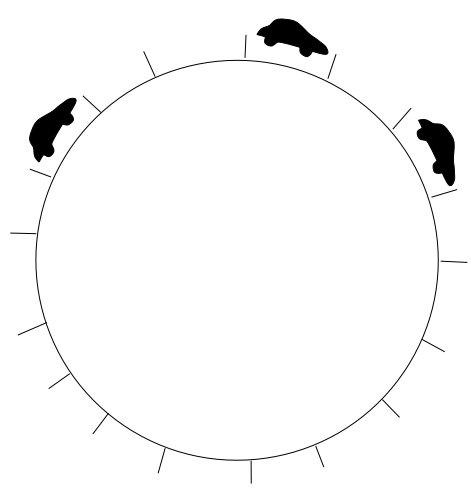

$4 \square>4$ 吕 


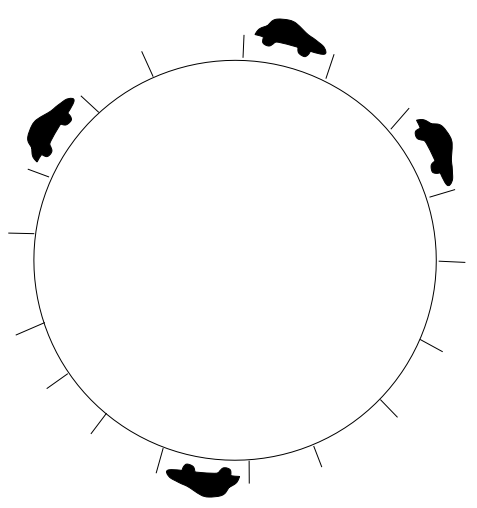

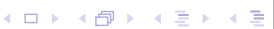




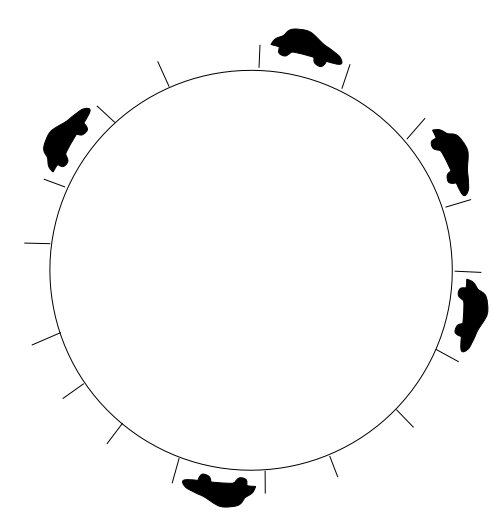


<smiles>C1CCCCCCCCCCCCCC1</smiles> 
Introduction

Main results

How many cars have burnt?

Formation of mesoscopic blocks
Forest fires

Knuth's parking scheme

Fires in the parking scheme

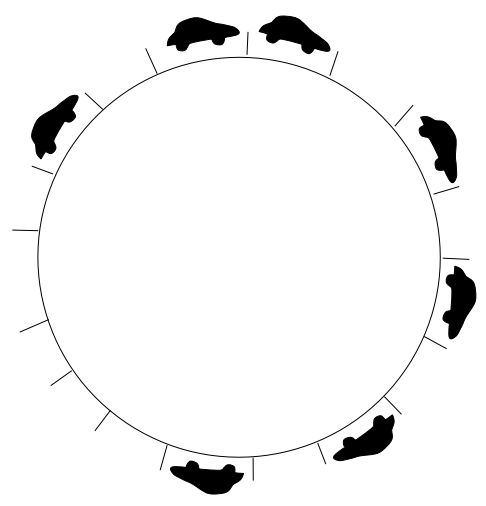

Jean BERTOIN

Burning cars in a parking lot 
Introduction

Main results

How many cars have burnt?

Formation of mesoscopic blocks
Forest fires

Knuth's parking scheme

Fires in the parking scheme

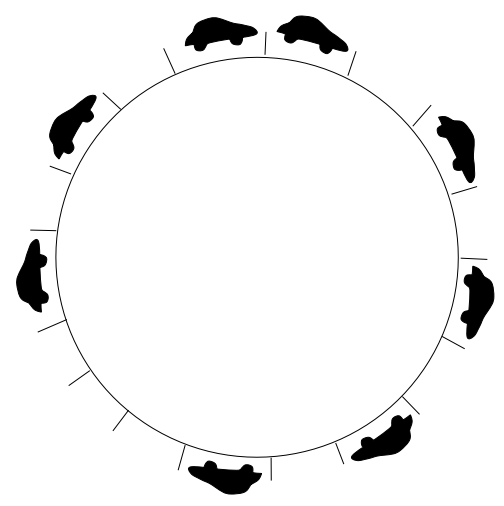

Jean BERTOIN

Burning cars in a parking lot 
If a car arrives at a site $j \in \mathbb{Z} / n \mathbb{Z}$ which is already occupied, then the car tries to park successively at sites $j+1, j+2, \ldots$ until it finally finds a vacant site. 

<smiles>C1CCCCCCCCCCCCCCC1</smiles> 
We incorporate fires to Knuth's parking model.

Molotov cocktails are thrown on the parking.

When a Molotov cocktail falls on an occupied site, the car parked there burns, the fire propagates to the neighboring cars, and the entire occupied block becomes instantaneously vacant. 


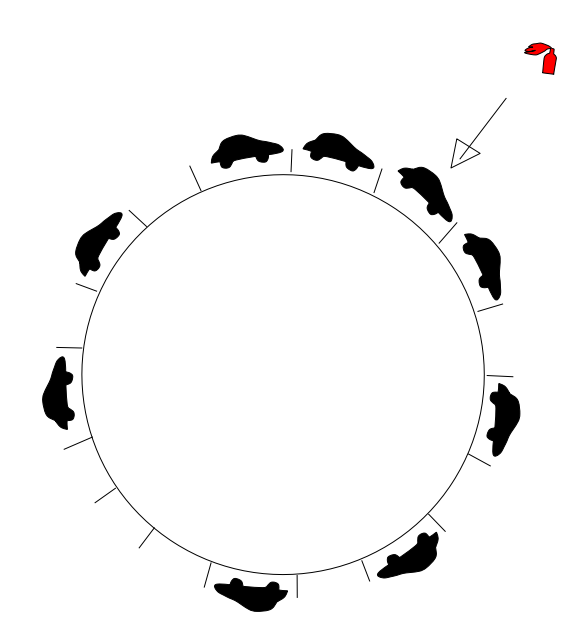


Introduction

Main results

How many cars have burnt?

Formation of mesoscopic blocks
Forest fires

Knuth's parking scheme

Fires in the parking scheme

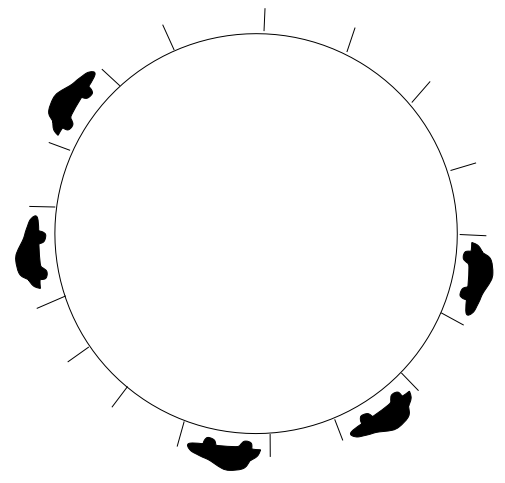

Jean BERTOIN

Burning cars in a parking lot 
The fundamental difference with the forest fire model on $\mathbb{Z} / n \mathbb{Z}$ is that a car may occupy a site different from that at which it arrived, whereas trees only grow on sites on which a seed falls.

The parking model thus involves a stronger spacial dependency. 
We assume that 
We assume that

- cars arrive independently on each site of $\mathbb{Z} / n \mathbb{Z}$ at a unit rate. 
We assume that

- cars arrive independently on each site of $\mathbb{Z} / n \mathbb{Z}$ at a unit rate.

- Molotov cocktails hit each site at rate $n^{-\alpha}$ for some parameter $\alpha \in(0,1)$, independently of car arrivals. 
We assume that

- cars arrive independently on each site of $\mathbb{Z} / n \mathbb{Z}$ at a unit rate.

- Molotov cocktails hit each site at rate $n^{-\alpha}$ for some parameter $\alpha \in(0,1)$, independently of car arrivals.

Denote the average occupation of the parking lot at time $t$ by

$$
\theta_{n}(t)=\frac{1}{n} \# \text { occupied sites at time } t
$$


Introduce the saturation time

$$
T_{n}=\inf \{t \geq 0: \text { all the sites are occupied }\}
$$


Introduce the saturation time

$$
T_{n}=\inf \{t \geq 0: \text { all the sites are occupied }\}
$$

When no Molotov cocktails are thrown, the parking becomes saturated when $n$ cars have arrived, i.e. at a time close to 1 . 
Introduce the saturation time

$$
T_{n}=\inf \{t \geq 0: \text { all the sites are occupied }\}
$$

When no Molotov cocktails are thrown, the parking becomes saturated when $n$ cars have arrived, i.e. at a time close to 1 .

\section{Do Molotov cocktails have a significant impact on the saturation of the parking?}




\section{Theorem}

(i) For every $\alpha>0$ and $t \in[0,1)$,

$$
\lim _{n \rightarrow \infty} \theta_{n}(t)=t \quad \text { in probability. }
$$

(ii) For $\alpha>2 / 3$,

$$
\lim _{n \rightarrow \infty} T_{n}=1 \text { in probability. }
$$

Thus for $\alpha>2 / 3$, the system becomes periodic as $n \rightarrow \infty$ : the parking lot gets filled almost as if there were no fire until complete saturation, and then becomes totally empty again after a Molotov cocktail has been thrown, destroying all the cars. 


\section{Theorem}

For $0<\alpha<2 / 3$, we have for every $t \in(1,2)$ that

$$
\lim _{n \rightarrow \infty} P\left(\theta_{n}(t) \leq t-1+\varepsilon\right)=1, \quad \forall \varepsilon>0
$$

while

$$
\lim _{n \rightarrow \infty} P\left(T_{n} \leq t\right)=0
$$


There is a phase transition at the critical parameter $\alpha=2 / 3$. 
There is a phase transition at the critical parameter $\alpha=2 / 3$.

- For $\alpha>2 / 3$, fires have a negligible impact on the saturation of the parking lot as saturation occurs at time $\approx 1$. 
There is a phase transition at the critical parameter $\alpha=2 / 3$.

- For $\alpha>2 / 3$, fires have a negligible impact on the saturation of the parking lot as saturation occurs at time $\approx 1$.

- For $\alpha<2 / 3$ the average occupation also grows almost linearly until time 1 and then drops suddenly to almost 0 , although the saturation time is not less than 2 . (in fact we conjecture that $T_{n} \rightarrow \infty$ in that case). 
The heart of the analysis consists in estimating $B_{n, m}$, the number of cars which have been burnt when the $m$-th car arrives, when both $m$ and $n$ are large: 
The heart of the analysis consists in estimating $B_{n, m}$, the number of cars which have been burnt when the $m$-th car arrives, when both $m$ and $n$ are large:

$$
E\left(B_{n, m}\right) \leq c \frac{n^{2-\alpha}}{n-m} .
$$

where $c$ is a numerical constant. 
When $\alpha>2 / 3$, we take $m \approx n-n^{\beta}$ for $2 / 3<\beta<\alpha$. So

$$
E\left(B_{n, m}\right) \leq c n^{2-\alpha} / n^{\beta} \ll n^{\beta},
$$

so with high probability, less than $n^{\beta}$ cars have been burnt when the $\left(n-n^{\beta}\right)$-th car arrives. 
When $\alpha>2 / 3$, we take $m \approx n-n^{\beta}$ for $2 / 3<\beta<\alpha$. So

$$
E\left(B_{n, m}\right) \leq c n^{2-\alpha} / n^{\beta} \ll n^{\beta},
$$

so with high probability, less than $n^{\beta}$ cars have been burnt when the $\left(n-n^{\beta}\right)$-th car arrives.

There are less than $2 n^{\beta}$ vacant sites.

The time needed for the arrival of $2 n^{\beta}$ new cars is $\approx 2 n^{\beta-1}$. 
When $\alpha>2 / 3$, we take $m \approx n-n^{\beta}$ for $2 / 3<\beta<\alpha$. So

$$
E\left(B_{n, m}\right) \leq c n^{2-\alpha} / n^{\beta} \ll n^{\beta},
$$

so with high probability, less than $n^{\beta}$ cars have been burnt when the $\left(n-n^{\beta}\right)$-th car arrives.

There are less than $2 n^{\beta}$ vacant sites.

The time needed for the arrival of $2 n^{\beta}$ new cars is $\approx 2 n^{\beta-1}$.

The probability that a Molotov cocktail is thrown during that time is $\approx n^{1-\alpha} \times 2 n^{\beta-1} \ll 1$. 
When $\alpha>2 / 3$, we take $m \approx n-n^{\beta}$ for $2 / 3<\beta<\alpha$. So

$$
E\left(B_{n, m}\right) \leq c n^{2-\alpha} / n^{\beta} \ll n^{\beta},
$$

so with high probability, less than $n^{\beta}$ cars have been burnt when the $\left(n-n^{\beta}\right)$-th car arrives.

There are less than $2 n^{\beta}$ vacant sites.

The time needed for the arrival of $2 n^{\beta}$ new cars is $\approx 2 n^{\beta-1}$.

The probability that a Molotov cocktail is thrown during that time is $\approx n^{1-\alpha} \times 2 n^{\beta-1} \ll 1$.

The parking lot is saturated when the $\left(n+n^{\beta}\right)$-th car arrives. 
The argument breaks down for $\alpha<2 / 3$ :

$4 \square \times 4$ 吕 
The argument breaks down for $\alpha<2 / 3$ :

The number of new cars arriving between two consecutive Molotov cocktails is $\approx n^{\alpha}$. 
The argument breaks down for $\alpha<2 / 3$ :

The number of new cars arriving between two consecutive Molotov cocktails is $\approx n^{\alpha}$.

We have $B_{n, m} \leq n^{\alpha}$ only for $n-m \geq n^{2(1-\alpha)}$. 
The argument breaks down for $\alpha<2 / 3$ :

The number of new cars arriving between two consecutive Molotov cocktails is $\approx n^{\alpha}$.

We have $B_{n, m} \leq n^{\alpha}$ only for $n-m \geq n^{2(1-\alpha)}$.

For such $m$, there are at least $n^{2(1-\alpha)}$ vacant sites. 
The argument breaks down for $\alpha<2 / 3$ :

The number of new cars arriving between two consecutive Molotov cocktails is $\approx n^{\alpha}$.

We have $B_{n, m} \leq n^{\alpha}$ only for $n-m \geq n^{2(1-\alpha)}$.

For such $m$, there are at least $n^{2(1-\alpha)}$ vacant sites.

Conclusion: since $n^{2(1-\alpha)} \gg n^{\alpha}$, further cars will be burnt during the time needed for the arrival of $n^{2(1-\alpha)}$ new cars. 
The argument breaks down for $\alpha<2 / 3$ :

The number of new cars arriving between two consecutive Molotov cocktails is $\approx n^{\alpha}$.

We have $B_{n, m} \leq n^{\alpha}$ only for $n-m \geq n^{2(1-\alpha)}$.

For such $m$, there are at least $n^{2(1-\alpha)}$ vacant sites.

Conclusion: since $n^{2(1-\alpha)} \gg n^{\alpha}$, further cars will be burnt during the time needed for the arrival of $n^{2(1-\alpha)}$ new cars. However this is not insufficient to establish rigorously the phase transition. 
The crucial step for $\alpha<2 / 3$ consists in establishing the existence of a small time window before 1 during which mesoscopic occupied intervals of size of order $n^{\beta}$ are formed for some $\beta>\alpha$. 
The crucial step for $\alpha<2 / 3$ consists in establishing the existence of a small time window before 1 during which mesoscopic occupied intervals of size of order $n^{\beta}$ are formed for some $\beta>\alpha$.

Since $n^{\beta-\alpha} \gg 1$, with high probability such mesoscopic intervals are entirely destroyed by a Molotov cocktail shortly after they have been formed.

This causes the average occupation to quickly drop to 0 . 
The crucial step for $\alpha<2 / 3$ consists in establishing the existence of a small time window before 1 during which mesoscopic occupied intervals of size of order $n^{\beta}$ are formed for some $\beta>\alpha$.

Since $n^{\beta-\alpha} \gg 1$, with high probability such mesoscopic intervals are entirely destroyed by a Molotov cocktail shortly after they have been formed.

This causes the average occupation to quickly drop to 0 .

During this short time interval, destruction of cars by fires overpasses significantly the arrivals of new cars, and thus prevents the saturation of the parking. 
The key technical issue is to estimate the mean of the number of cars which have been burnt when the $m$-th car arrives: for $m<n$

$$
E\left(B_{n, m}\right) \leq c n^{2-\alpha} /(n-m) .
$$


The key technical issue is to estimate the mean of the number of cars which have been burnt when the $m$-th car arrives: for $m<n$

$$
E\left(B_{n, m}\right) \leq c n^{2-\alpha} /(n-m) .
$$

We need an upperbound for the mean damage done by Molotov cocktails that have been thrown between the arrival of the $j$-th and the $(j+1)$-th car. Then we sum for $j=0$ to $n-m$. 
The key technical issue is to estimate the mean of the number of cars which have been burnt when the $m$-th car arrives: for $m<n$

$$
E\left(B_{n, m}\right) \leq c n^{2-\alpha} /(n-m) .
$$

We need an upperbound for the mean damage done by Molotov cocktails that have been thrown between the arrival of the $j$-th and the $(j+1)$-th car. Then we sum for $j=0$ to $n-m$.

Recall that the probability that a Molotov cocktail is thrown between the arrival of the $j$-th car and the arrival of the $(j+1)$-th car is $n^{-\alpha}$. 
Further, given that a Molotov cocktail is thrown, it arrives at a site chosen uniformly at random, and thus destroyed one of the occupied blocks picked at random with a size bias, i.e. the probability for a given occupied block to be destroyed is proportional to its size. 
Further, given that a Molotov cocktail is thrown, it arrives at a site chosen uniformly at random, and thus destroyed one of the occupied blocks picked at random with a size bias, i.e. the probability for a given occupied block to be destroyed is proportional to its size.

We are led to bound from above

$$
E\left(\beta_{n, j}^{2}\right) / E\left(\beta_{n, j}\right)
$$

where $\beta_{n, j}$ denotes one of the occupied blocks after the arrival of the $j$-th car chosen uniformly at random. 
A genuine difficulty in the analysis of fire models if the lack of monotonicity

However some rough comparisons are useful. 
A genuine difficulty in the analysis of fire models if the

\section{lack of monotonicity}

However some rough comparisons are useful.

Let us couple two systems having the same process of car arrivals, the first involving fires due to Molotov cocktails and the second not. 
A genuine difficulty in the analysis of fire models if the

\section{lack of monotonicity}

However some rough comparisons are useful.

Let us couple two systems having the same process of car arrivals, the first involving fires due to Molotov cocktails and the second not.

Then the occupation of the parking lot is less in the first model than in the second.

In particular, if we compare occupied blocks picked at random with a size-bias after the arrival of the $j$-th car, then we get a smaller quantity in the model with fire than in the model without fires. 
Knuth parking scheme (without fires) has been studied in depth by Chassaing, Louchard, Flajolet, Pittel and others. Right after the arrival of the $j$-car, there remain $n-j=k$ vacant sites, viewed as left-extremities of occupied blocks. 
Knuth parking scheme (without fires) has been studied in depth by Chassaing, Louchard, Flajolet, Pittel and others. Right after the arrival of the $j$-car, there remain $n-j=k$ vacant sites, viewed as left-extremities of occupied blocks.

The sequence of spacing between consecutive vacant sites has the same distribution as a sequence of $k$ i.i.d. Borel(s) variables conditioned on having sum $n$.

$$
P(\beta(s)=\ell)=\frac{\mathrm{e}^{-s \ell}(s \ell)^{\ell-1}}{\ell !}, \quad \ell \geq 1 .
$$

The parameter $s$ can be chosen arbitrarily in $(0,1]$ (due to the conditioning on the total sum). 
The first and second moments of $\beta(s)$ are

$$
\mathbb{E}(\beta(s))=\frac{1}{1-s} \quad \text { and } \quad \mathbb{E}\left(\beta(s)^{2}\right)=\frac{1}{(1-s)^{3}} .
$$


The first and second moments of $\beta(s)$ are

$$
\mathbb{E}(\beta(s))=\frac{1}{1-s} \quad \text { and } \quad \mathbb{E}\left(\beta(s)^{2}\right)=\frac{1}{(1-s)^{3}} .
$$

Informally, conditioning the sum of $k=n-j$ i.i.d. Borel(s) variables to have sum equal to $n$ forces

$$
(n-j) \mathbb{E}(\beta(s))=\frac{n-j}{1-s}=n
$$

i.e.

$$
\frac{1}{1-s}=\frac{n}{n-j} .
$$


Then

$$
\frac{E\left(\beta_{n, j}^{2}\right)}{E\left(\beta_{n, j}\right)} \approx \frac{1}{(1-s)^{2}} \approx\left(\frac{n}{n-j}\right)^{2}
$$


Then

$$
\frac{E\left(\beta_{n, j}^{2}\right)}{E\left(\beta_{n, j}\right)} \approx \frac{1}{(1-s)^{2}} \approx\left(\frac{n}{n-j}\right)^{2} .
$$

Returning to our initial problem of estimating the number of cars that have been burnt before the arrival of the $m$-th car, we conclude that

$$
E\left(B_{n, m}\right) \leq n^{-\alpha} \sum_{j=0}^{m-1}\left(\frac{n}{n-j}\right)^{2} \approx \frac{n^{2-\alpha}}{n-m}
$$


To conclude, we just give the idea of the proof of the formation of mesoscopic occupied blocks in the case $\alpha<2 / 3$. Specifically, we have to show that we can find $m(n) \sim n$, such that at the arrival time of the $m(n)$-th car, the size of the occupied block containing a site picked uniformly at random is greater than $n^{\beta}$ with high probability for some $\beta>\alpha$. 
To conclude, we just give the idea of the proof of the formation of mesoscopic occupied blocks in the case $\alpha<2 / 3$. Specifically, we have to show that we can find $m(n) \sim n$, such that at the arrival time of the $m(n)$-th car, the size of the occupied block containing a site picked uniformly at random is greater than $n^{\beta}$ with high probability for some $\beta>\alpha$. Molotov cocktails are thrown on an interval of size $n^{\beta}$ at rate $n^{\beta-\alpha} \gg 1$ and thus such mesoscopic interval are destroyed shortly after their formation. 
Again we use a coupling with Knuth's parking (i.e. without fires). 
Again we use a coupling with Knuth's parking (i.e. without fires).

We first consider Knuth's parking model and establish that for $m \approx n-n^{\gamma}$ with $2 / 3<\gamma<1$, the size of the occupied block containing a site picked uniformly at random is $\asymp n^{2-2 \gamma}$ with high probability. 
Again we use a coupling with Knuth's parking (i.e. without fires).

We first consider Knuth's parking model and establish that for $m \approx n-n^{\gamma}$ with $2 / 3<\gamma<1$, the size of the occupied block containing a site picked uniformly at random is $\asymp n^{2-2 \gamma}$ with high probability.

Then we incorporate damages due to Molotov cocktails. We have already obtained the bound $\mathrm{Cn}^{2-\alpha-\gamma}$ for the mean number of cars which have been burnt. 
In short, one has to check that the fires have not altered too much occupied blocks in Knuth's model. 
In short, one has to check that the fires have not altered too much occupied blocks in Knuth's model.

More precisely, one shows that for $\varepsilon>0$, with high probability when $n$ is sufficiently large, an occupied block of size $\ell n^{2-2 \gamma}$ for Knuth's model contains an occupied block with size at least $(1-\varepsilon) \ell n^{2-2 \gamma}$ in the model with Molotov cocktails added provided that

$$
\frac{2}{3} \vee(1-2 \alpha / 3)<\gamma<1 .
$$

We take $\beta=2-2 \gamma$, and then $\beta>\alpha$ provided $\alpha<2 / 3$. 
This requires a connexion between parking schemes and certain periodic paths which was already used in works by Chassaing, Flajolet and Louchard. 